dent both before and after $\mathrm{GuCl}$ treatment. Consideration of the unfolding and refolding processes involved suggests that even a minimally altered $\omega$ region may affect the in vitro folding of other regions or domains of the enzyme in a way different from the in vivo situation. It must be acknowledged that these experiments were carried out on crude extracts of enzymes and mutants. If, however, they were reproduced using purified systems like those of Langley et al., they would pose most interesting and potentially productive questions about the kinetic pathways along which large polypeptide chains fold in the test tube on the one hand and off the ribosome on the other.

\section{Mössbauer effect and order-disorder transitions in alloys}

from G. Longworth

To study the mechanisms involved in order-disorder reactions in alloys, the functional dependence of some physical quantity (for example resistance, magnetisation or X-ray diffraction pattern) must be measured. An ordered alloy is one whose structure may be divided into sublattices each of which is occupied predominantly by one type of atom, whereas after disordering the various atoms are arranged at random on each sublattice. Such reactions are divided into those of first or second order (degree) involving either a discontinuous or continuous change in long-range order parameter with temperature. At a first order transition, usually associated with closely packed lattices such as in $\mathrm{Cu}_{3} \mathrm{Au}$ or $\mathrm{Ni}_{3} \mathrm{Fe}$, two phases of different composition may coexist, whose transformation is governed by a latent heat. The highly monochromatic gamma rays emitted by certain (Mössbauer) nuclides may be used to study order-disorder reactions by measuring the hyperfine interactions, which are a function of local atomic order.

Thus the complex ordering in $\mathrm{Fe}_{3} \mathrm{Al}$ has been studied by Cser et al. (Phys. Stat. Sol., 20, 581; 1967) by determining the changes in magnetic hyperfine field $\left(H_{\text {hf }}\right)$ at the ${ }^{57} \mathrm{Fe}$ sites. In iron alloys, $H_{\mathrm{hf}}$ is determined essentially by the magnetic moment on the iron atom and by the conduction electron polarisation, both of which may be a function of local atomic order. Erickson and Roberts (Phys. Rev., B9, 9; 1974) have measured the change on ordering in the electron charge density at the gold nuclei, and in the vibrational motion of the gold atoms in $\mathrm{Cu}_{3} \mathrm{Au}$.
Drijver et al. (J. Phys., Paris, 35, C6-465; 1974) have used an analysis of the hyperfine fields in $\mathrm{Ni}_{3} \mathrm{Fe}$ to determine the long range order parameter, and more recently (Phys. Rev. Lett., 34, $16 ; 1975)$ have extended this technique to follow the time evolution of the phase transition in this alloy 'at temperature'. A detailed analysis of the Mössbauer lineshapes confirmed that the transition was first order, proceeding by way of a nucleation and growth mechanism.

In this latest work, a sharp drop in mean field $H_{\mathrm{hf}}$ at the iron sites at about $771 \mathrm{~K}$ on heating indicates a first order transition, while during cooling, the reverse path is followed with a hysteresis of about $10 \mathrm{~K}$. The linewidths $(\Gamma)$ at the transition were much greater than the theoretical value $\left(0.20 \mathrm{~mm} \mathrm{~s}^{-1}\right)$, mainly due to the presence of both ordered and disordered regions having values of $H_{\text {hf }}$ which differed by a small amount ( $\simeq 20$ koersted). The amount of ordered material at $770.9 \mathrm{~K}$ roughly follows the increase in the mean field $\bar{H}_{\mathrm{hr}}$ with time, along an S-shaped curve, consistent with nucleation and growth, taking about 200 hours to complete. By comparison with model calculated spectra, it was suggested that: (1) during the ordering reaction the first nuclei present have a long range order parameter of only about 0.6 . There is then a homogenous growth of order within these ordered segregates. (2) During the disordering reaction there is in addition composition segregation involving iron-rich ordered material and iron-poor disordered material consistent with a two-phase region about $4 \mathrm{~K}$ wide similar to that observed by Calvayrac et al. (Mater. Res. Bull., 7, 891 ; 1972) using electron microscopy. While disordering starts at a temperature at or above the two-phase region, ordering is suppressed as shown by the hysteresis and starts about $10 \mathrm{~K}$ below.

The disadvantage of the Mössbauer technique is that for detailed measurements above room temperature it is really restricted to iron and possibly tin alloys, since for most other Mössbauer nuclides the size of the Mössbauer effect is too small. In the case of $\mathrm{Ni}_{3} \mathrm{Fe}$ the amount of information available from the spectra is limited by the relatively small difference in $H_{\mathrm{hf}}$ between the ordered and disordered phase. One might expect a larger difference when a change in crystal structure is also involved. The use of small amounts of the Mössbauer nuclide ${ }^{119} \mathrm{Sn}$ as a probe in magnetic alloys might lead to a greater change in $H_{\mathrm{hf}}$, since the tin atoms carry no magnetic moment and derive their field entirely from their neighbours. Thus the Mössbauer technique is re- stricted in its application in this field. But the advantage of being able to measure the changes in local properties at the temperatures concerned is considerable, and this technique, combined ideally with electron microscopy, could add greatly to our knowledge of order-disorder transitions in alloys.

\section{Analysis of membrane noise}

from Shin-Ho Chung

A NUMBER of researchers are using a powerful new technique to study electrical properties of nerve and muscle membranes. This technique, known as membrane noise analysis, is based on the fact that spontaneous perturbations of the mechanisms underlying membrane permeability give rise to random fluctuations in membrane conductance, and the statistical structure of these fluctuations provides, in turn, informaion about the microscopic events generating such noise.

On the basis of physical theory, electrical noise arising from several different sources is expected to be present in nerve and muscle membranes. First, the thermal agitation of charge carriers will produce thermal, or Nyquist, noise. In addition, when the flow of current is due to only a relatively small number of the total available carriers (as it is in the membrane), flicker noise is shown to be present. Superimposed on these two sources, there will be random fluctuations of membrane conductance resulting from microscopic perturbation of ionic channels. For example, single ionic channels in a membrane at an equilibrium state may spontaneously open and close, although the average number of open channels remains constant. The membrane conductance will then fluctuate around its mean value. $\mathrm{Be}$ cause each of these noise sources has a different physical basis, their analysis, in principle at least, will provide answers to different sets of questions which have not previously been amenable to experimental attack. An experiment by Conti, De Felice and Wanke (J. Physiol., Lond., 248, 45-82: 1975) was aimed at elucidating some of these questions.

Two physical models for ionic channels follow from Hodgkin and Huxley's description of current flows in membranes. The first of these, a probabilistic version of the Hodgkin-Huxley equations, envisages a channel gate, guarded by four macromolecules, either in an open or closed conformation. The channel is open when all these macromolecules are in their open conformation. An alternative class of 